\title{
Developing Sigmoid Volvulus after Medical Disimpaction of a Patient with Stool Impaction: A Case Report
}

\author{
Alharthi $\mathrm{A}^{* 1}$ and Almarwani $\mathrm{M}^{2}$ \\ ${ }^{1}$ Consultant Internist FHAFH Jeddah, Kingdom of Saudi Arabia \\ ${ }^{2}$ Consultant Invasive Radiologist KFAFH Jeddah, Kingdom of Saudi Arabia
}

*Corresponding author: Alharthi A, Consultant Internist FHAFH Jeddah, Kingdom of Saudi Arabia, E-mail: dr.adilkfafh@hotmail.com

Citation: Alharthi A, Almarwani M (2019) Developing Sigmoid Volvulus after Medical Disimpaction of a Patient with Stool Impaction: A Case Report. J Case Rep Stud 7(4): 403. doi: 10.15744/2348-9820.7.403. doi: 10.15744/2348-9820.7.403

Received Date: July 25, 2019 Accepted Date: August 26, 2019 Published Date: August 28, 2019

\begin{abstract}
Stool impaction is an ailment resulted from chronic constipation which commonly found in the elderly population. It causes wide range of potential complications if not promptly diagnosed and treated. These complications doubled when elder people experience other co- morbidities. A case of an 80 years old lady with advanced dementia, bedridden and non-communicating is presented for clinical study. This patient who was presented with abdominal distention and changes in her mental status was found to have stool impaction based on the clinical and radiological test conducted to her. After ten days of proximal softening with distal washout therapy (using polyethylene glycol through PEG tube and lactulose enema), her condition deteriorated as her abdominal distention increased. An abdominal X-ray and CT scan showed the occurrence of sigmoid volvulus which wasn't there previously. She underwent decompression colonoscopy procedure successfully. The records, observations, and procedures conducted in this case recounts that proximal softening with distal washout therapy pushed the stool to the redundant sigmoid colon of this elder patient, resulting in twisting of the colon that caused sigmoid volvulus. The study also presents discussion about the complications of stool impaction, the complication of enemas, relevant published cases, and guidelines of palliative constipation management in the elderly population.
\end{abstract}

Keywords: Fecal; Medical Impaction; Sigmoid Volvulus; Stool Impaction

\section{Introduction}

Stool impaction is defined as the development of a large, dry colonic mass of stools that prevents the proper defecation process. The stool may pile up and reach the highest level of the proximal colon, if the problem is not noticed and corrected. The primary site of impaction is by far the rectum followed by the sigmoid colon [1]. Proper recognition of this problem is important for its prevention that can only be done after the appreciation of the groups at risk, who are the cognitively impaired and immobilized. This is more common among the older population suffering from dementia; although, it is also common in younger populations with mental retardation. A systemic review concluded that the elderly, underlying neuropsychiatric disease and hospitalized or institutionalized patient are within the high risk group in which fecal impaction must be suspected [2]. The above patient group reduced water intake, reduce gastrointestinal transit time (functional age wise vs. pathological or medication induced), and also reduce overall mobility contributes towards the pathogenesis of stool impaction [3].

\section{Cases Presentation}

An 80 years old lady was noticed by her daughter to be behaving differently in her mental status for the last week. She was already known to have dementia and was bedridden. She was being fed by percutaneous endoscopic gastrostomy (PEG) tube and breathing by tracheostomy tube. She was unable to communicate and was totally dependent on her family for her activities of daily living. The daughter did not notice fever, vomiting, or any difficulties in feeding her by the PEG tube. However, she did notice constipation that was worsening in the last 2 weeks and was manifested as hard frequent small stools. Clinically, the patient was breathing comfortably. Moreover, she was awake but not aware about the surrounding. Her temperature was $36.8{ }^{\circ} \mathrm{C}$, blood pressure was $110 / 70 \mathrm{~mm} \mathrm{Hg}$, with $95 \%$ saturation of ambient air. The abdomen of the patient was distended, non-tender, soft, and hypertempanic. PEG tube insertion site was clean without signs of infection. The rectal digital examination of hard stools and preliminary labs came out to be normal, but the abdominal X-ray presentation revealed that the hardened stools filling the descending and ascending colon, with proximal colon dilation, distal colon and rectal collapse (Figure 1). The patient was admitted as a case of stool impaction, and she was given polyethyl glycol (2 sachets in $800 \mathrm{ml}$. of water given over 5-6 hours) through the PEG tube once and lactulose enemas for 3 days. Initially, she passed large stools; however, the abdomen remained distended. 
Therefore, she was prescribed another cycle of the same medications. For the 2 days post medications - 10 days post presentationshe did not pass stool. Her abdomen was more distended and digital rectal examination failed to find stool. CT scan abdomen showed radiological signs of sigmoid volvulus e.g whirlpool sign and birds beak sign (Figures 2 and 3), and no radiological signs of bowel ischemia (Figure 4). The patient was diagnosed with sigmoid volvulus. Gastroenterology teams were consulted, and she underwent an emergency decompression colonoscopy. The team successfully performed the procedure to the patient.

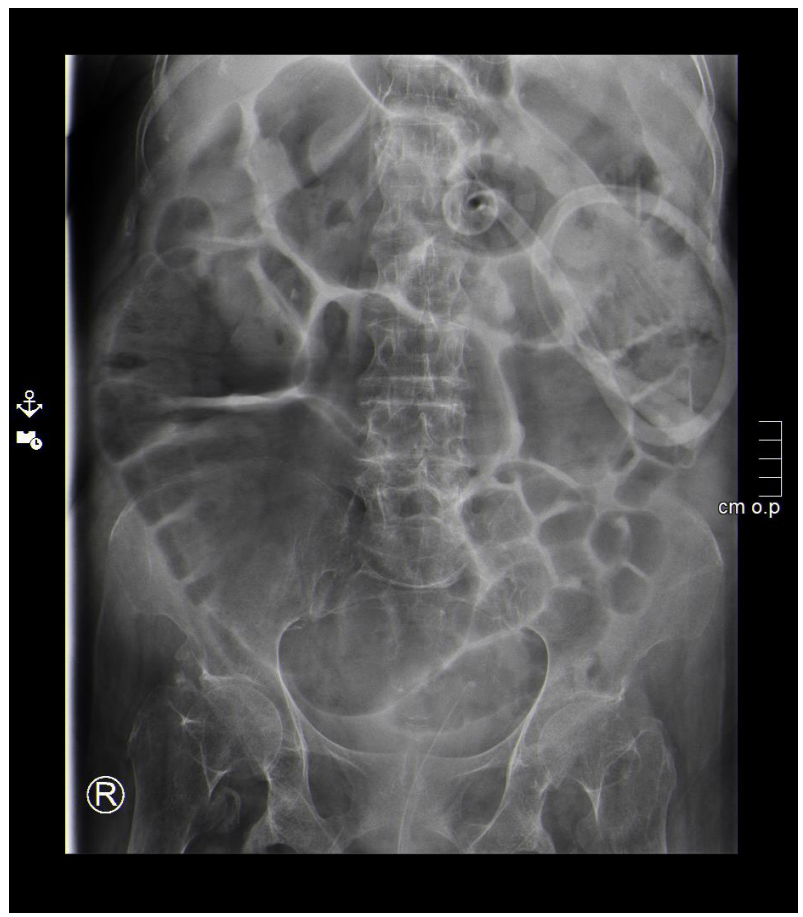

Figure 1: Abdominal radiograph showing dilated large bowl with no clear air in the rectum - done upon presentation

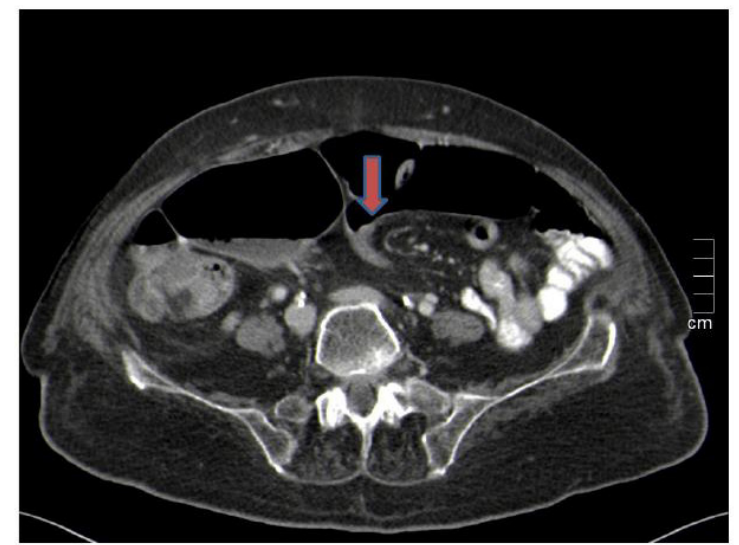

Figure 2: CT image showing the afferent loop of the distal descending cool twists and narrows as it enters the volvulus (arrow) with twisting of the mesentery and mesentric vessels (whirlpool sign) - done 10 days post presentation

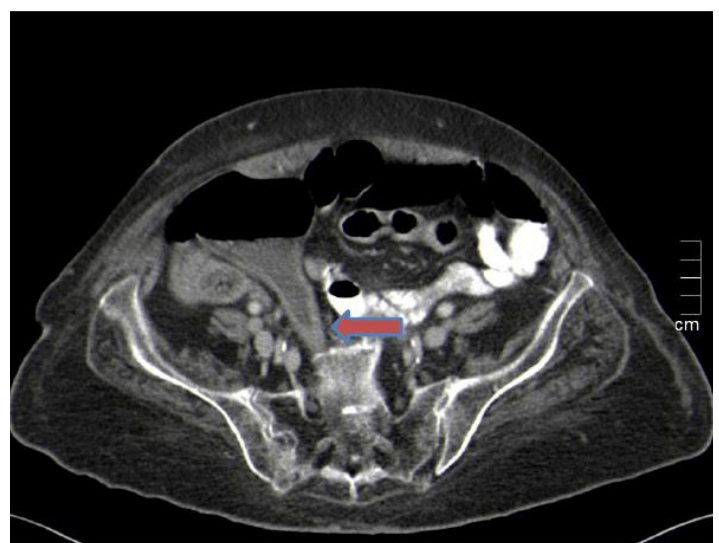

Figure 3: CT image showing the afferent loop of the distal sigmoid colon twisting as it exists the volvulus giving the (Birds beak sign) at the transitional point with the rectum (arrow) -done 10 days post presentation 


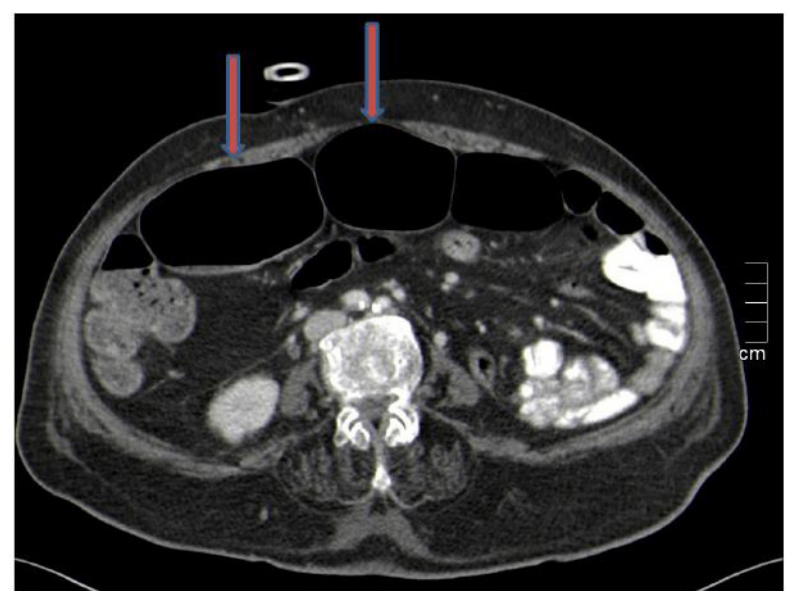

Figure 4: CT image showing the dilated sigmoid colon (arrow) which has no wall thickening, no surrounding fat stranding, no free fluid, no pneumatosis intestinalis (no signs of bowel ischemia/ compromise) - done 10 days post presentation

\section{Discussion}

In the above case it is important to clarify that the sigmoid volvulus had not developed initially before the impaction treatment; however, it is most likely a complication of it. There is no such similar case, despite of this logically suspected complication of the impaction treatment. It is also important to clarify that the above polyethyl glycol therapy given for proximal stool impaction is contraindicated in mechanical obstruction. It shouldn't be given in patients with ongoing abdominal cramps or nausea, which was difficult to anticipate in our patient who was not communicating due to advanced dementia. Nonetheless, practitioners should be extra cautious about this medication in such non- communicating frail patient to prevent possible complications. As the patient had no hemodynamic compromise, no concerning changes in her lab results, and no CT signs of ischemic bowel (Figure 4), so there was no absolute indication for surgery, equally important is the patient's frailty and weak general condition that implies intolerance to major surgery and general anesthesia. The Gastroenterology team found it more appropriate to go for emergency colonic decompression.

In a retrospective study including 168 patients nonoperative decompression as the initial treatment was successful in more than $90 \%$ of cases; however recurrence rate was high as well. The study concluded that nonoperative decompression could be suggested as the sole treatment for the first episode of volvulus [4]. Another study found that endoscopic detorsion had a good success rate as well and argued that other advantages are proper assessment of colonic viability [5]. The assessment of stool impaction should be multidisciplinary as part of the comprehensive geriatric assessment, which includes the review of full co-morbidities and medications taken by the patient and their relation to stool impaction pathogenesis, associated medical problems such as electrolyte imbalance metabolic and endocrine disorders. These disorders need to be identified and appropriate palliative solutions should be taken by the multidisciplinary team [6]. Management for this disease is mainly based on enema for rectal impaction and proximal softening medications for higher up impaction with complications. The physicians need to be aware of proper anticipation and assessment of this fragile age group [7]. A similar study presented a case of 73-year-old man, who was paraplegic and known to have chronic constipation. He was presented with abdominal pain and abdominal distention. His abdominal X-ray showed a large stool impaction. Therefore, he received rehydration and multiple enemas with some benefit and ultimately underwent an elective surgery of the redundant megacolon [8]. Another case study presented a 76-year-old female with bilateral lower limbs swelling. Her abdomen was distended without tenderness associated with marked bilateral edema of the legs. Computed tomography (CT) scan showed gross dilatation of the colon and rectum representing stool impaction and bilateral pelvic vein thrombosis. Moreover, Doppler ultrasonography confirmed a thrombosis of the external iliac vein and common femoral veins on both sides [9]. A similar case was presented of a 43-year-old man without significant abdominal history who was presented with abdominal pain and absence of stool for one week. CT abdomen showed signs of fecal impaction and sigmoiditis. Initial conservative treatment was converted to surgical exploration within 24 hours because of clinical deterioration. Laparotomy revealed a complete sigmoid volvulus and the presence of a large feacalith $[10,11]$.

\section{Conclusion}

Stool impaction is a common condition of the immobilized elder patients with potential serious consequences. It can be prevented by properly screening of high-risk population. This study has discussed about the complications of stool impaction, the complication of enemas, relevant published cases, and guidelines of palliative constipation management in the elderly. The study results have clarified that sigmoid volvulus had not developed initially before the impaction treatment; however, it is most likely a complication of it. Author also discourages from giving polyethyl glycol to patient such as ours to prevent possible complications. 


\section{Acknowledgements}

The author is very thankful to all the associated personnel in any reference that contributed in/for the purpose of this research. Further, this research holds no conflict of interest and is not funded through any source. Abstract presented at following conference report: (Adil Alharthi et al., J Clin Case Rep 2017, 7:8(Supp) DOI :10.4172/2165-7920-C1-012).

\section{Funding}

This research did not receive any specific grant from funding agencies in the public, commercial, or not-for-profit sectors.

\section{Author's Contribution}

Alharthi A conceived the study idea, conducted the online reference search and prepared the Article for linguistics. Almarwani M chose the appropriate images and the figure captions. Both authors have critically reviewed and approved the final draft and are responsible for the content and similarity index of the manuscript.

\section{References}

1. Constipation Remedies for all (2012) Dealing with fecal impaction A frustrating and sometimes dangerous Complication of constipation, France.

2. Serrano Falcón B, Barceleo Lopes M, Beatriz Mateos B, Alvarez Sanchez A, Ray E (2016) "Fecal impaction: a systematic review of its medical complications. BMC Geriatr 16: 4.

3. Araghizadeh F (2005) Fecal impaction. Clin Colon Rectal Surg 18: 116-9.

4. Johansson N, Rosemar A, Angenete E (2018) Risk of recurrence of sigmoid volvulus: a single-centre cohort study. Colorectal Dis 20: 529-35.

5. Iida T, Nakagaki S, Satoh S, Shimizu H, Kaneto H, et al. (2017) Clinical outcomes of sigmoid colon volvulus: identification of the factors associated with successful endoscopic detorsion. Intest Res 15: 215-20.

6. Larkin PJ, Sykes NP, Centeno C, Ellershaw JE, Elsner F, et al. (2008) The management of constipation in palliative care: clinical practice recommendations. Palliat Med 22: 796-807.

7. Ramakrishnan K, Scheid D (2003) Enemas: A "Purge" Atory. The Inter J Gastroenterol IJGE/3/1/10503.

8. Di Saverio S, Tugnoli G, Orlandi PE, Casali M, Catena F, et al. (2009) A 73-year-old man with long-term immobility presenting with abdominal pain. PLoS Med 6: e1000092.

9. Dewulf M, Blomme Y, Coucke C (2018) Faecal impaction causing bilateral pelvic venous thrombosis. Acta Chir Belg 118: 110-2.

10. Colebunders B, Philipsen TE, Van der Stighelen Y (2009) Sigmoid Volvulus Caused by a Large Faecalith. Case Report. Acta Chir Belg 109: 504-6.

11. Hussain ZH, Whitehead DA, Lacy BE (2014) Fecal Impaction. Curr Gastroenterol Rep 16: 404.



